\title{
Role of Sentinel Lymph Node in Early Stage of Uterine Cervical Cancer
}

\author{
Mihaela Mădălina Gavrilescu ${ }^{1}$, Ana-Maria Todosi ${ }^{1}$, Nicolae loanid ${ }^{2 *}$ and Viorel Scripcariu ${ }^{1}$ \\ ${ }^{1}$ Department of Surgery, Gr. T. Popa University of Medicine and Pharmacy lași, Romania \\ ${ }^{2}$ First Unit of Oncologic Surgery, Regional Cancer Institute Iași, Romania
}

\begin{abstract}
Cervical cancer is currently the second most common form of neoplasia worldwide and third in the female population. Dissemination can occur directly (isthmus, parametrium, vagina, urinary bladder and/or rectum), through the Iymphatic system (parametrium, internal iliac, external iliac, common iliac, obturator lymph nodes and rarely in the inferior gluteus, superior gluteus, superior rectum, sacrum, aortic lymph nodes) and through the circulatory system (lung, mediastinum, bones, liver). The risk of pelvic lymph nodes invasion in stage IB (FIGO) is $9-17 \%$. The standard surgical treatment, for stages IA2-IIA, is radical hysterectomy with pelvic lymphadenectomy. The risk of intraoperative (vessel or nerve damage) or postoperative complications (lymphedema) is not negligible. The sentinel node concept refers to the first lymph node in which the cancerous lymphatic drainage takes place. This idea has radically changed the therapeutic approach in the treatment of breast cancer and melanoma. In cervical cancers, this technique is $92 \%$ accurate with only an $8 \%$ false negative rate. Currently, the sentinel node protocol is not included in the standard treatment for cervical cancer because certain issues need to be addressed (the sensitivity of the frozen section examination, the pathologist's subjectivity, the uniformity of the protocol, the surgical experience, the size of the tumor).
\end{abstract}

Keywords: Uterine Cervical Cancer; Sentinel Node; Radical Hysterectomy; Neoadjuvant Treatment

\section{Introduction}

Cervical cancer is currently the second most common form of neoplasia worldwide and third in the female population. It is the first or second most common cancer (after breast cancer) in less developed countries. Cervical cancer accounted for approximately 275,000 deaths worldwide in 2008 [1]. The incidence rates of invasive cervical cancer per 100,000 women in different geographical areas largely vary, reflecting differences in HPV infection and access to screening [2].

Radical hysterectomy with pelvic lymphadenectomy is the standard treatment for early stage cervical cancer. The goal of a systematic lymphadenectomy is to identify and remove tumor cells transported to the lymphatic tissue draining the uterine cervix and the upper vagina.

The term "sentinel lymph node" was first used in 1960 [3], but the initial results on the success of sentinel node hypothesis were largely misinterpreted.

The concept of sentinel lymph node was introduced in 1977 by Cabanas for penile neoplasm. In 1992, Morton took this idea and demonstrated the veracity of sequential lymphatic invasion in cutaneous malignant melanoma [4]. In 1993, Giuliano and Krag initiated the first researches for assessing the sentinel node in breast cancer and provided information on therapeutic management in the early stages of disease [5]. When neoplastic dissemination by lymphatic embolization occurs, invasion is initially in the first tumordraining lymph node. This lymph node was called sentinel node. Identification, excisional biopsy, histopathology and immunohistochemistry allow to assess regional nodal status and to determine if regional lymphadenectomy is indicated or not [6].

Sentinel lymph node is the first lymph node in a nodal basin to drain an anatomically well-defined region and its histologic assessment is representative for all the other lymph nodes in that region.
This concept can be used particularly in patients diagnosed with cervical cancer stages IA to IIB, with a low incidence of lymph nodes invasion. Morbidity associated with complete pelvic lymphadenectomy (vessel or nerve damage, lymphedema) can be avoided [7-9].

\section{Sentinel Node Technique}

Sentinel node technique is by minimally invasive approach and its goal is to detect the first lymph node to drain the primary tumor and if the sentinel node is not invaded to avoid complete regional lymphadenectomy. Sentinel node technique uses radioactive tracers or vital dyes that are injected around the tumor and trace the route of tumor cells detached from the primary tumor to the first draining lymph node. Excisional biopsy and histopathological examination of the sentinel node are useful for selecting the patients requiring complete pelvic lymphadenectomy is required.

The technique using vital dye is easier and is performed intraoperatively. Marking with dye can be done using several types of dyes, of which the most used are: isosulfan blue, patent blue, methylene blue, China ink, indigo carmine, indocyanine green. Shortly after dye injection, the stained lymphatic channels which lead to sentinel node are visualized. The time window for sentinel node detection is short because the vital dye washes out rapidly.

*Corresponding author: Nicolae loanid, Str. General Berthlot No 2-4, First Unit of Oncologic Surgery, Regional Cancer Institute, 700483, lasi, Romania, Tel: +40(0)740 619 863; E-mail: n_ioanid@yahoo.com

Received: June 14, 2014, Accepted: July 10, 2014, Published: July 15, 2014

Citation: Citation: Gavrilescu MM, Todosi AM, loanid N, Scripcariu V. Role of Sentinel Lymph Node in Early Stage of Uterine Cervical Cancer. Journal of Surgery [Jurnalul de Chirurgie] 2014; 10(3): 217-222. doi:10.7438/1584-9341-10-3-3

Copyright: () 2014 Gavrilescu MM, et al. This is an open-access article distributed under the terms of the Creative Commons Attribution License, which permits unrestricted use, distribution, and reproduction in any medium, provided the original author and source are credited. 
The radioactive method consists in the preoperative injection of a radioactive tracer, preoperative scintigraphy, and the use of intraoperative gamma probe. It implies a high cost, requires specific technical equipment, and interdisciplinary collaboration. The most commonly used radioactive agent is technetium- $99 \mathrm{~mm}$ metastable colloidal sulfide $(\mathrm{Tc}-99 \mathrm{~m})$, which has the advantage of rapidly enhancing the regional nodes and washes out after several hours.

Scintigraphy is useful as it identifies the location of sentinel node and indicates the incision site, the gamma radiation detection probe intraoperatively identifying the radioactive lymph nodes.

Sentinel lymph node is noted as follows: $\mathrm{pNx}$ (sn): sentinel node cannot be assessed; pN0 (sn): no sentinel node metastasis; pN1 (sn): sentinel node metastases [10].

Based on the encouraging results in patients with vulva, penile, and breast cancer and in some cases of malignant melanoma, sentinel node technique was introduced for patients with cervical cancer in 1999, when several procedures were reported: in some studies sentinel node biopsy was performed by laparotomy or laparoscopy [11].

\section{Sentinel Lymph Node Concept in Gynecologic Disorders}

Sentinel lymph node biopsy is the preferred method for assessing axillary nodal histopathologic status in patients with breast cancer stages I and II. This technique has been recommended following the publication of the results of several recent randomized clinical trials showing the low morbidity in breast cancer patients performing sentinel node biopsy versus axillary lymph node dissection. However, this approach is not recommended by the American Society of Clinical Oncology (ASCO) in the following situations: large or locally advanced breast tumors (T3 and T4), signs of inflammatory breast cancer, tumor diagnosed during pregnancy, or the presence of palpable axillary lymph nodes. NCCN (National Comprehensive Cancer Network) guidelines recommend sentinel node biopsy in patients diagnosed with breast cancer without clinically evident axillary lymph nodes [12].

In breast cancer, the identification of sentinel nodes with a dyebased technique is considered inferior to gamma radiation technique. Cox et al. reported a sentinel node identification rate of $80.3 \%$ with the dye technique, $88.6 \%$ with gamma radiation method, and $96.7 \%$ when both techniques were used [13]. Kim et al. reported a detection rate of $83.1 \%, 89.2 \%$ and $91.9 \%$ for the dye method, $\gamma$ radiation-based technique, and combined technique, respectively, with a false-negative rate of $10.9 \%, 8.8 \%$, and $7.0 \%$, respectively [14].

In a study of 374 breast cancer patients in which sentinel node biopsy was performed using dye (indigo carmine, 2-4 mL injected around the primary tumor and periareolarly), followed by surgery with radical intent, the sentinel node was identified in 362 patients (96.8\%). In 54 patients sentinel node metastases were identified for which axillary lymph node dissection was performed. Sensitivity in detecting lymph node metastasis in final histopathology was $96.4 \%$ with a specificity of $100 \%$. The accuracy of sentinel node biopsy for the detection of metastatic disease was $98.1 \%$. The intraoperative diagnosis of sentinel node metastasis was confirmed in 362 patients by final histopathology, with a false-negative rate of $12.9 \%$ (8 patients) [15]. Vulvar cancer is a good target for sentinel node detection because the tumor can be easily injected with radiocolloid substances or vital dye and the lymphatic drainage is predictable in one or both inguinal areas.
In a study by Levenback et al. on a series of 452 patients diagnosed with vulvar cancer, in 418 at least one sentinel node was identified. Sensitivity of sentinel node testing was $91.7 \%$ [16].

Hauspy et al. reported in a study of 41 patients with vulvar cancer stages I and II in which following sentinel node assessment a complete inguinofemoral lymphadenectomy was performed, a detection rate for at least 1 sentinel lymph node per patient of 95\%, and bilateral sentinel nodes detected in $46 \%$ of patients [17].

Another study evaluated postoperative morbidity in patients with vulvar cancer who underwent sentinel node biopsy (29 patients) versus inguinal sentinel lymph nodes dissection (46 patients). The reported results showed that inguinal sentinel lymph node dissection was associated with a shorter operation time, a reduced rate of inguinal seromas, wound breakdown and infection, fewer days of inguinal drainage, and reduced postoperative lymphatic secretion [18].

\section{Sentinel Lymph Node Cervical Cancer}

Dissemination can occur directly (isthmus, parametrium, vagina, urinary bladder and/or rectum), through the lymphatic system (parametrium, internal iliac, external iliac, common iliac, obturator lymph nodes and rarely in the inferior gluteus, superior gluteus, superior rectum, sacrum, aortic lymph nodes) and through the circulatory system (lung, mediastinum, bones, liver). The incidence of pelvic lymph node invasion varies with FIGO stages. The literature reports an incidence of $11-21 \%$ in stage IB and of $39-43 \%$ in stage IIB. The incidence of paraaortic lymph nodes invasion ranges from $2-4 \%$ stage IB to $7-17 \%$ in stage IIB $[19,20]$. In stage I cervical cancer the risk of lymph node dissemination depends on tumor size, depth of stromal invasion, and presence of lymph-vascular space invasion.

In stage I cervical cancer the presence of lymph node invasion is the most important prognostic factor. If lymph node metastases are present at the time of surgery, the 5-year survival rate decreases from $85 \%$ to $50 \%[21,22]$. The alternative explorations of nodal status in cervical cancer (including imaging techniques) were disappointing.

Cervical cancer is a type of cancer in which sentinel node technique can be used for several reasons; literature data report an incidence of lymph node metastasis of $0-16 \%$ in patients with cervical tumors not exceeding $2 \mathrm{~cm}$ and of $15-31 \%$ in patients with FIGO stage IB [23] anatomically, uterine cervix is characterized by a complex lymphatic drainage, the following lymph node stations being described:

Station I includes the lymph node groups located anterior to a transverse plane crossing the bifurcation of the common iliac vessels, represented by: Lucas-Championniere lymph nodes (near the junction of uterine vessels and ureter), internal and external iliac nodes. During station I lymph node dissection it is important to recognize and remove the following lymph nodes: Cuneo-Marcille (situated at the bifurcation of the iliac vessels) and obturator nodes described by Thoma Ionescu and Leveuf-Godard (actually belonging to the medial external iliac lymph node chain, located between the inferior medial margin of external iliac vein and obturator nerve) [24]

- Station II, represented by the common iliac, promontoral, and lateral and presacral lymph nodes

- Station III, represented by inframesenteric aortic lymph nodes

- Station IV, intrarenal aortic lymph nodes. 
In 2010, Cibula et al. reported a proposal for a new classification system of types of pelvic lymph node dissection. The four types are:

- Type sentinel lymph node: only the sentinel lymph nodes detected in the pelvic basin are removed.

- Type I dissection: external iliac region: removes lymph nodes anterior and medial to the external iliac vessels, ventrally up to the deep circumflex iliac vein. Obturator region: removes obturator nodes above the obturator nerve. Common iliac region: removes

nodes anterior (superficial common iliac region) up to the mid common iliac vessels.

- Type II dissection: this is a type I dissection which also includes all of the following steps:

External iliac region: removes nodes between the external iliac vessels and psoas muscle after vessel mobilization and complete skeletonization; removes distal nodes caudal to deep circumflex iliac vein.

Internal iliac region: exposes the internal iliac vein and removes internal iliac nodes. Obturator region: removes nodes below obturator nerve.

Presacral region: complete removal of presacral nodes.

Type III dissection: type II dissection which also includes all of the following steps:

Common iliac region: removal of superficial common iliac nodes up to the aortic bifurcation;

Complete medialization of the common iliac vessels and removal of deep common iliac nodes between the lateral aspect of the common iliac vein and the psoas muscle exposing lumbosacral trunk [25].

The literature shows a one-side sentinel node detection rate of over $90 \%$ (range $55 \%$ to $100 \%$ ). This detection rate is not influenced by the surgical approach (laparotomy or laparoscopy). Also, bilateral sentinel node was detected at a rate ranging from 31\% [26] to 94\% (Pijpers et al., 2004) [27].

In 2000, Medl et al. were the first to report the sentinel node concept in cervical cancer. They studied only 3 patients in which sentinel node was identified by using a blue substance during radical hysterectomy. In all 3 cases the sentinel node was positive [28]. Another study conducted at the University of Texas on 21 patients identified the sentinel node in $60 \%$ of them by injecting isosuflan bluebased substance into the cervix [29].

In 2003, Dargent et al. recommended laparoscopic sentinel node biopsy for cervical cancer staging and determining the therapeutic management: surgical or neoadjuvant treatment [30].

Recently, some authors have evaluated preoperative and intraoperative lymphoscintigraphy for sentinel node detection (detection rate $84-100 \%$ ) [7]. Verheijen et al. [31] used a combined technique (vital dye and lymphoscintigraphy) in a group of 10 patients and reported an $80 \%$ sentinel node detection rate in cervical cancer.

A systematic review of the literature on the benefit of sentinel node biopsy in the assessment of lymph node status in cervical cancer and which technique is best (vital dye, Tc $-99 \mathrm{~m}$ or combined method) was conducted and the results were presented in an article that included 842 patients. Data were collected from international databases (Medline and Embase).
Finally, 23 studies were selected with a sentinel node detection rate of $97 \%$ for the combined approach versus $84 \%(\mathrm{p}<0.0001)$ for dye and $88 \%(\mathrm{p}<0.0018)$ for Tc $-99 \mathrm{~m}$ [32].

Optimization of sentinel node technique refers to the use of method that is inexpensive, feasible for cervical cancer patients, and has a high detection rate. Colorimetric technique based on the peritumoral injection of 2-4 ml methylene blue 10-360 minutes preoperatively was studied in 77 patients who underwent abdominal radical hysterectomy and pelvic lymphadenectomy. Results of the study showed that methylene blue is a useful tracer to detect sentinel nodes the ideal dose being $4 \mathrm{ml}$ injected 60-90 minutes prior to surgery [33].

In the assessment of lymph node status in patients diagnosed with cervical cancer by the use of sentinel node technique, regardless of the method, colorimetric, radioisotopic or combined, laparoscopy plays an important role in determining the subsequent treatment [34-36].

Another study provided information on radical surgery, emphasizing that parametrial resection can be minimized in patients with cervical tumors and negative sentinel node. Inclusion criteria for the study group were: patients with stage IAl cervical cancer and lymphovascular space invasion, IB1 with tumor size below $2 \mathrm{~cm}$ and stromal invasion detected by imaging below $50 \%$. The 60 patients underwent laparoscopic sentinel node mapping. All patients with positive nodes also had positive sentinel node [37].

Like many other studies in the literature, Roy et al. compared the two methods of sentinel node detection in cervical cancer: colorimetric method with isosulfan blue and radioisotopic method with Tc-99. The study included 211 patients with early stage cervical cancer, in which surgery was performed laparoscopically to identify and remove the sentinel node, followed by systematic pelvic lymphadenectomy with or without paraaortic lymph node sampling. The colorimetric method was used in 152 patients, and the radioisotopic method in 166 patients. The authors concluded that sentinel node mapping using the radioisotopic technique had a higher detection rate (96.9\%) compared with the use of dye (92.8\%). The most frequent locations of the sentinel node (85\%) were the bifurcation of the iliac vessels and/or obturator fossa. It should be mentioned that complete pelvic lymphadenectomy can be performed in patients in whom sentinel nodes were not detected [38].

In order to minimize the need for pelvic lymphadenectomy, sentinel node technique seems to be a good option for assessing nodal status in early stage cervical cancer. A prospective study by Cormier et al. based on colorimetric and radioisotopic techniques reported similar results on sentinel node detection with those previously published in the literature. At least one sentinel lymph node was identified in $93 \%$ of cases (114 of the 122 study patients), while optimal (bilateral) mapping was achieved in $75 \%$ of cases (91/122). Moreover, the authors believe that this procedure accompanied by sentinel node ultrastaging is an effective technique to increase the detection rate of lymph node metastases by $3.5 \%$ [39].

The studies recommend the use of sentinel node technique in early stage cervical cancer.

\section{Where to Look for the Sentinel Node?}

Bader et al. reported that in cervical cancer patients with only one positive node, this is most frequently located in the external iliac artery and obturator fossa or parameterium. Thus, sentinel node should be first looked for in these regions. If only one node is positive, the location of the other invaded nodes is hard to predict. However, dissemination of tumor cells to paraaortic lymph nodes is rare [40]. 
Di Stefano et al. in a study on a series of 50 patients with FIGO stage IA2 - IIA cervical cancer concluded that sentinel node detection using vital dye is a feasible procedure with important role in surgical staging of patients with small cervical tumors. The described method was based on dye injection 5-10 mm into the cervix at 3, 6, 9, 12 hours (dye injection directly into the tumor was avoided). Once the abdomen was opened, blue lymph nodes were detected in the pelvis in no less than 15 minutes and no more than 70 minutes the reported results indicated a sentinel node detection rate of $90 \%$, unilateral and bilateral sentinel nodes being detected in $72 \%$ and $60 \%$ of the cases, respectively.

Topographically, sentinel nodes were found in the external iliac artery in $55 \%$ of cases and obturator fossa in $38 \%$ of cases, with only 5 sentinel nodes isolated in the common iliac region [23].

Similar results were published and Diaz et al. in a prospective study that included 81 patients with early stage cervical cancer (FIGO stages IA1-IIA) with indication for surgical treatment. All patients received intraoperative injection of vital dye. Sixty-three patients who underwent preoperative lymphoscintigraphy also received a $99 \mathrm{~m} \mathrm{Tc}$ sulfur colloid injection and imaging assessment one hour after the injection at tumor periphery in all four quadrants of uterine cervix. Sentinel node mapping was successful in 77 (95\%) of the 81 patients. The total number of sentinel nodes evaluated was 316 , with an average of 3 nodes per patient. The three locations of sentinel node were: external iliac (35\%), internal iliac (30\%), and obturator fossa $(20 \%)$ [41].

Sentinel lymph node mapping in early stage cervical cancer (IA1 IIA) was once again a topic debated by Darlin et al. in a study on 105 patients. The technique described for obtaining the results was based on radioactive tracer injection $(1.5 \mathrm{~mL}$ of Tc99 nanocolloid human albumin) in the four cervical peritumoral quadrants 18 hours before surgery, dynamic scintigraphy (15 minutes after tracer injection up to 45-60 minutes), and use of intraoperative gamma probe. During surgery (robot assisted laparoscopic approach-da Vinci Surgical System in 90 patients, laparotomy or laparoscopy in the remaining 25 patients) the detection rate of at least one sentinel node was $90 \%$ (94/105 patients), and $94 \%$ in patients with tumor size equal or less than $2 \mathrm{~cm}$. Bilateral nodes were detected in 62 of the 105 patients. The authors concluded that sentinel node technique may be useful in patients with cervical tumors not exceeding $2 \mathrm{~cm}$. However, complete pelvic lymphadenectomy has to be performed in cases with unilaterally positive sentinel node [42].

\section{Diagnostic Accuracy of Sentinel Node}

The question is whether sentinel node has significance in locally advanced cervical cancer. Barranger et al. gave some answers in a study on 33 patients, 23 with early stage cervical cancer who underwent complete laparoscopic pelvic lymphadenectomy after sentinel node assessment (combined vital dye- radioisotope) and 10 with locally advanced cervical cancer who underwent laparoscopic pelvic and para-aortic lymphadenectomy. The results of this study showed that sentinel nodes were detected in $86.9 \%$ of early-stage cervical cancer cases and in $80 \%$ of the locally advanced cervical cancer cases. Since the cervix is a middle organ in the pelvis, another important aspect approached by the study was the unilateral or bilateral involvement of the sentinel node. It is therefore impossible to predict the preferential side of lymphatic drainage in these cases. The false-negative rate of sentinel node biopsy was 0 in the early stage group and $20 \%$ in the group with locally advanced cervical cancer.
When the side of dissection was analyzed, the false-negative rate reached $42.9 \%$ in patients with locally advanced cancer and remained 0 in the early-stage group $(\mathrm{p}=0.038)$ [43].

Many authors report, in addition to sentinel node detection rate, the false-negative rate (defined as negative sentinel node but presence of neoplastic dissemination to other nodes in the region), which seems to be the most important performance index.

A retrospective multicenter study evaluated the false-negative rate of sentinel lymph node ultrastaging in patients with FIGO stages I-IIB cervical cancer. 645 patients were included in the study, and sentinel lymph nodes were detected bilaterally in $72 \%$ of cases and unilaterally in only $28 \%$. The authors reported a sensitivity of the technique (using either vital dye alone or in combination with radioisotope) of $97 \%$ with a false-negative rate of $1.3 \%$ for patients with bilaterally detected sentinel nodes. The most important contribution of sentinel lymph ultrastaging in the management of cervical cancer is to improve detection of lymph node metastases, including micrometastases (deposits of 0.2 to $2 \mathrm{~mm}$ ) and isolated tumor cells (deposits not exceeding $0.2 \mathrm{~mm}$, including the presence of single, non-cohesive cytokeratin-positive tumor cells) [44].

Another challenge that arises in many studies is that of complete lymphadenectomy in cervical cancer and why sentinel node seems to be a better solution in the pretherapeutic staging of these patients. Therefore, a study by Gortzak-Uzan et al. provided information about node metastatization and therapeutic management of early stage cervical cancer. The results showed that lymph node metastasis detection rate was two-times higher when using the sentinel node technique as compared to complete lymphadenectomy. The authors believe there are two explanations: one attributed to extensive histological examination (ultrastaging) of sentinel node, and the second explanation is that lymph node metastases in sentinel node procedures are related to aberrant pathways and unusual locations (e.g., common iliac or paraaortic regions). Since postoperative morbidity can be reduced and the negative rate is quite low in many studies, sentinel node procedure is adequate for assessing lymph node status in early stage cervical cancer [45].

\section{Discussions}

Lymph node involvement in cervical cancer is the main prognostic factor. Lymph node assessment can be done by imaging methods before treatment, but the data are insufficient or inaccurate; therefore, the most accurate method is pelvic lymphadenectomy, but it is also associated with complications described in the literature. Sentinel node concept has become the topic of many studies aimed at investigating the feasibility of this technique in early cervical cancer.

Lymphatic mapping and sentinel node identification are procedures used in almost all solid tumors, and sentinel node status has become part of the staging criteria in both breast cancer and malignant melanoma.

At the present time, sentinel lymph node is still investigational and not standard of care in cervical cancer. The overall sentinel lymph node identification is around $90 \%$, with a sensitivity of $92 \%$, and an approximately $8 \%$ false negative rate, with a negative predictive value of $97 \%$. Challenges to adoption include the sensitivity of frozen section, availability of pathologic expertise, uniformity of technique, surgical experience, and clinical impact of tumor size [46]. 


\section{Conclusion}

Sentinel lymph node biopsy is a technique that can be used to assess nodal status in early stage cervical cancer patients, mentioning that complete pelvic lymphadenectomy minimizes postoperative morbidity. A fast and accurate image of the lymph nodes allows the correct selection of therapeutic approach: concomitant neoadjuvant chemoradiotherapy or radical hysterectomy.

However, there are questions that need answers and optimal results for the introduction of sentinel node procedure in cervical cancer treatment. For example, which is the acceptable false-negative rate? What does the learning curve for this technique involve? If the sentinel

node is negative, is it oncologically safe to abandon pelvic lymphadenectomy? What is the role of micrometastases and isolated tumor cells in pelvic recurrences?

\section{Conflict of interest}

The authors have no conflicts of interest to disclose.

\section{Acknowledgements}

The corresponding author is a $\mathrm{PhD}$ student at the "Gr. T. Popa" University of Medicine and Pharmacy Iasi. This paper is the result of research during the doctoral internship within the project Interuniversity partnership for increasing the medical doctoral quality and interdisciplinary through doctoral scholarships - DocMed.net POSDRU/107/1.5/S/78702.

The authors thank AMPOSDRU for supporting the research for this study.

\section{References}

1. Ferlay J, Shin HR, Bray F, Forman D, Mathers C, et al. (2010) Estimates of worldwide burden of cancer in 2008: GLOBOCAN 2008. Int J Cancer 127: 2893-2917.

2. Arbyn M, Castellsagué X, de Sanjosé S, Bruni L, Saraiya M, et al. (2011) Worldwide burden of cervical cancer in 2008. Ann Oncol 22: 2675-2686.

3. Gould Ea, Winship T, Philbin Ph, Kerr Hh (1960) Observations On A "Sentinel Node" In Cancer Of The Parotid. Cancer 13: 77-78.

4. Morton DL, Wen DR, Wong JH, Economou JS, Cagle LA, et al. (1992) Technical details of intraoperative lymphatic mapping for early stage melanoma. Arch Surg 127: 392-399.

5. Krag DN, Weaver DL, Alex JC, Fairbank JT (1993) Surgical resection and radiolocalization of the sentinel lymph node in breast cancer using a gamma probe. Surg Oncol 2: 335-339.

6. Blidaru A, Bordea C, Voinea S, Condrea I, Albert P, et al. (2006) Validation protocol of sentinel node biopsy for breast cancer using radioactive tracer at the Institute of Oncology Bucharest "Prof. Dr. Alexandru Trestioreanu" Chirurgia 101: 391-399.

7. Angioli R, Palaia I, Cipriani C, Muzii L, Calcagno M, et al. (2005) Role of sentinel lymph node biopsy procedure in cervical cancer: a critical point of view. Gynecol Oncol 96: 504-509.

8. Barranger E, Cortez A, Uzan S, Callard P, Darai E (2004) Value of intraoperative imprint cytology of sentinel nodes in patients with cervical cancer. Gynecol Oncol 94: 175-180.

9. Buist MR, Pijpers RJ, van Lingen A, van Diest PJ, Dijkstra J, et al. (2003) Laparoscopic detection of sentinel lymph nodes followed by lymph node dissection in patients with early stage cervical cancer. Gynecologic oncology 90: 290-296.

10. Tehnica ganglionului santinela
11.

Di Stefano AB, Acquaviva G, Garozzo G, Barbic M, Cvjeticanin B, et al. (2005) Lymph node mapping and sentinel node detection in patients 12. with cervical carcinoma: a 2-year experience. Gynecol Oncol 99: 671-679. Kwak HY, Chae BJ, Bae JS, Kim EJ, Chang EY, et al. (2013) Feasibility of sentinel lymph node biopsy in breast cancer patients clinically suspected of axillary lymph node metastasis on preoperative imaging. World J Surg 13. Oncol 11: 104.

Cox CE, Bass SS, McCann CR, Ku NN, Berman C, et al. (2000) Lymphatic mapping and sentinel lymph node biopsy in patients with 14. breast cancer. Annu Rev Med 51: 525-542.

Kim T, Giuliano AE, Lyman GH (2006) Lymphatic mapping and sentinel lymph node biopsy in early-stage breast carcinoma: a metaanalysis.

15. Cancer 106: 4-16.

Takamaru T, Kutomi G, Satomi F, Shima H, Ohno K, et al. (2014) Use of the dye-guided sentinel lymph node biopsy method alone for breast

cancer metastasis to avoid unnecessary axillary lymph node dissection.

16. Exp Ther Med 7: 456-460.

Levenback CF, Ali S, Coleman RL, Gold MA, Fowler JM, et al. (2012) Lymphatic mapping and sentinel lymph node biopsy in women with squamous cell carcinoma of the vulva: a gynecologic oncology group 17. study. J Clin Oncol 30: 3786-3791.

Hauspy J, Beiner M, Harley I, Ehrlich L, Rasty G, et al. (2007) Sentinel

18. lymph node in vulvar cancer. Cancer 110: 1015-1023.

Hefler LA, Grimm C, Six L, Seebacher V, Polterauer S, et al. (2008) Inguinal sentinel lymph node dissection vs. complete inguinal lymph node dissection in patients with vulvar cancer. Anticancer Res 28:

19. 515-517

Daraï E, Rouzier R, Ballester M, Barranger E, Coutant C (2008) Sentinel lymph node biopsy in gynaecological cancers: the importance of 20. micrometastases in cervical cancer. Surg Oncol 17: 227-235.

Levenback CF (2007) Status of sentinel lymph nodes in cervical cancer.

21. Gynecol Oncol 107: S18-19.

Rob L, Pluta M, Strnad P, Hrehorcak M, Chmel R, et al. (2008) A less radical treatment option to the fertility-sparing radical trachelectomy in

22. patients with stage I cervical cancer. Gynecol Oncol 111: S116-120.

Altgassen C, Hertel H, Brandstädt A, Köhler C, Dürst M, et al. (2008) Multicenter validation study of the sentinel lymph node concept in 23. cervical cancer: AGO Study Group. J Clin Oncol 26: 2943-2951.

Di Stefano AB, Acquaviva G, Garozzo G, Barbic M, Cvjeticanin B, et al. (2005) Lymph node mapping and sentinel node detection in patients

24. with cervical carcinoma: a 2-year experience. Gynecol Oncol 99: 671-679. Coleman R LC (2004) Lymphatics of the cervix. In: Levenback C, van der ZeeAGJ, Coleman RL (Eds) Clinical lymphatic mapping in gynecologic 25. cancers. Taylor and Francis. London and New York

Cibula D, Abu-Rustum NR. (2010) Pelvic lymphadenectomy in cervical cancer--surgical anatomy and proposal for a new classification system.

26. Gynecol Oncol 116: 33-37.

Lantzsch T, Wolters M, Grimm J, Mende T, Buchmann J, et al. (2001)

Sentinel node procedure in Ib cervical cancer: a preliminary series. $\mathrm{Br} \mathrm{J}$ 27. Cancer 85: 791-794.

Pijpers R, Buist MR, van Lingen A, Dijkstra J, van Diest PJ, et al. (2004) The sentinel node in cervical cancer: scintigraphy and laparoscopic gamma probe-guided biopsy. Eur J Nucl Med Mol Imaging 31: 1479-1486.

Medl M, Peters-Engl C, Schütz P, Vesely M, Sevelda P (2000) First report of lymphatic mapping with isosulfan blue dye and sentinel node biopsy 29. in cervical cancer. Anticancer Res 20: 1133-1134.

O'Boyle JD, Coleman RL, Bernstein SG, Lifshitz S, Muller CY, et al. (2000) Intraoperative lymphatic mapping in cervix cancer patients undergoing radical hysterectomy: A pilot study. Gynecol Oncol 79: 238-243. 
30. Dargent D, Enria R (2003) Laparoscopic assessment of the sentinel lymph nodes in early cervical cancer. Technique--preliminary results and future developments. Crit Rev Oncol Hematol 48: 305-310.

31. Malur S, Krause N, Köhler C, Schneider A (2001) Sentinel lymph node detection in patients with cervical cancer. Gynecol Oncol 80: 254-257.

32. van de Lande J, Torrenga B, Raijmakers PG, Hoekstra OS, van Baal MW, et al. (2007) Sentinel lymph node detection in early stage uterine cervix carcinoma: a systematic review. Gynecol Oncol 106: 604-613.

33. Yuan SH, Xiong Y, Wei M, Yan XJ, Zhang HZ, et al. (2007) Sentinel lymph node detection using methylene blue in patients with early stage cervical cancer. Gynecol Oncol 106: 147-152.

34. Lambaudie E, Collinet P, Narducci F, Sonoda Y, Papageorgiou T, et al. (2003) Laparoscopic identification of sentinel lymph nodes in early stage cervical cancer: prospective study using a combination of patent blue dye injection and technetium radiocolloid injection. Gynecol Oncol 89: 84-87.

35. Kushner DM, Connor JP, Wilson MA, Hafez GR, Chappell RJ, et al. (2007) Laparoscopic sentinel lymph node mapping for cervix cancer--a detailed evaluation and time analysis. Gynecol Oncol 106: 507-512.

36. Coutant C, Morel O, Delpech Y, Uzan S, Daraï E, et al. (2007) Laparoscopic sentinel node biopsy in cervical cancer using a combined detection: 5-years experience. Ann Surg Oncol 14: 2392-2399.

37. Strnad P, Robova H, Skapa P, Pluta M, Hrehorcak M, et al. (2008) A prospective study of sentinel lymph node status and parametria involvement in patients with small tumour volume cervical cancer. Gynecol Oncol 109: 280-284.

38. Roy M, Bouchard-Fortier G, Popa I, Grégoire J, Renaud MC, et al. (2011) Value of sentinel node mapping in cancer of the cervix. Gynecol Oncol 122: 269-274.
39. Cormier B, Diaz JP, Shih K, Sampson RM, Sonoda Y, et al. (2011) Establishing a sentinel lymph node mapping algorithm for the treatment of early cervical cancer. Gynecol Oncol 122: 275-280.

40. Bader AA, Winter R, Haas J, Tamussino KF (2007) Where to look for the sentinel lymph node in cervical cancer. Am J Obstet Gynecol 197: 678

41. Diaz JP, Gemignani ML, Pandit-Taskar N, Park KJ, Murray MP, et al. (2011) Sentinel lymph node biopsy in the management of early-stage cervical carcinoma. Gynecol Oncol 120: 347-352.

42. Darlin L, Persson J, Bossmar T, Lindahl B, Kannisto P, et al. (2010) The sentinel node concept in early cervical cancer performs well in tumors smaller than $2 \mathrm{~cm}$. Gynecol Oncol 117: 266-269.

43. Barranger E, Coutant C, Cortez A, Uzan S, Darai E (2005) Sentinel node biopsy is reliable in early-stage cervical cancer but not in locally advanced disease. Ann Oncol 16: 1237-1242.

44. Cibula D, Abu-Rustum NR, Dusek L, Slama J, Zikán M, et al. (2012) Bilateral ultrastaging of sentinel lymph node in cervical cancer: Lowering the false-negative rate and improving the detection of micrometastasis. Gynecol Oncol 127: 462-466

45. Gortzak-Uzan L, Jimenez W, Nofech-Mozes S, Ismiil N, Khalifa MA, et al. (2010) Sentinel lymph node biopsy vs. pelvic lymphadenectomy in early stage cervical cancer: is it time to change the gold standard? Gynecol Oncol 116: 28-32.

46. Frumovitz M, Ramirez PT, Levenback CF (2008) Lymphatic mapping and sentinel lymph node detection in women with cervical cancer. Gynecol Oncol 110: S17-20. 